\title{
Effects of a single bout of exercise on mitochondria-mediated apoptotic signaling in rat cardiac and skeletal muscles
}

\author{
Su-Zi Yoo', Mi-Hyun No', Jun-Won Heo', Eunwook Chang', Dong-Ho Park', Ju-Hee Kang², Dae-Yun Seo', Jin Han³, Su-Jeen Jung', \\ Kwan Hwangbo ${ }^{5}$, Hyo-Bum Kwak ${ }^{1, *}$ \\ 'Department of Kinesiology, Inha University, Incheon, Korea \\ ${ }^{2}$ Department of Pharmacology and Medicinal Toxicology Research Center, Inha University School of Medicine, Incheon, Korea \\ ${ }^{3}$ National Research Laboratory for Mitochondrial Signaling, Department of Physiology, College of Medicine, Cardiovascular and Metabolic Disease Center, Inje University, \\ Busan, Korea \\ ${ }^{4}$ Department of Leisure Sports, Seoil University, Seoul, Korea \\ ${ }^{5}$ Technical \& Education Office, Korea Football Association, Seoul, Korea
}

This study aimed to determine the effects of a single bout exercise on mitochondria-mediated apoptotic signaling in cardiac and skeletal muscles. Fischer 344 rats (4 months old) were randomly divided into the control or a single bout of exercise group $(n=10$ each). The rats performed a single bout of treadmill exercise for $60 \mathrm{~min}$. Mitochondria-mediated apoptotic signaling (e.g., $\mathrm{Bax}, \mathrm{Bcl}-2$, mitochondrial permeability transition pore [mPTP] opening, cytochrome $c$, and cleaved caspase-3) was measured in cardiac (e.g., left ventricle) and skeletal (e.g., soleus and white gastrocnemius) muscles. A single bout of exercise signifi- cantly decreased mPTP opening sensitivity in all tissues. However, a single bout of exercise did not show any statistical differences in Bax, Bcl-2, cytochrome $c$, and cleaved caspase-3 in all tissues measured. A single bout of exercise did not show definite results on characteristics of mitochondria-mediated apoptotic signaling. Therefore, further research is necessary to provide a more mechanistic understanding of the apoptosis pathway.

Keywords: Apoptosis, Exercise, Mitochondria, Heart, Skeletal muscle

\section{INTRODUCTION}

Mitochondria are the powerhouses responsible for the energy production that occurs in cells called adenosine triphosphate (ATP) synthesis (Murphy et al., 2016). In addition to providing cellular energy, mitochondria are involved in various tasks, such as cellular respiration, oxidative stress, and calcium homeostasis. Mitochondria also conduct mitochondrial quality control; (a) maintaining mitochondrial morphology, integrity, number, and size through cycles of mitochondrial dynamics (fusion and fission); (b) it promotes the turnover of damaged mitochondria and inhibits the accumulation of dysfunctional mitochondria through mitochondrial autophagy (mitophagy) (Yoo et al., 2019).
Mitochondria can serve as mediators, coordinating a highly programmed cell death referred to as apoptosis (Redza-Dutordoir and Averill-Bates, 2016). According to previous studies, dysfunctional mitochondria and mitochondrial reactive oxygen species (mtROS) induce the initial events of mitochondria-mediated apoptosis by releasing pro-apoptotic proteins into the cytoplasm (Redza-Dutordoir and Averill-Bates, 2016; Xiong et al., 2014). Imbalance between pro-apoptotic proteins (e.g., Bax and Bid) and antiapoptotic proteins (e.g., Bcl-2 and $\mathrm{Bcl}-\mathrm{xl}$ ) induces the opening of the mitochondrial permeability transition pore (mPTP) and the release of cytochrome $\mathrm{c}$ which serves as an electron transporter in the electron transfer chain of inner mitochondrial membranes. The mitochondria-released cytochrome c binds to ATP, apoptotic protease-acti-

\footnotetext{
*Corresponding author: Hyo-Bum Kwak (D) https://orcid.org/0000-0003-0451-4554 Department of Kinesiology, Inha University, 100 Inha-ro, Michuhol-gu, Incheon 22212, Korea 
vating factor 1 , and pro-caspase 9, which activates caspase-3, consequently resulting in DNA fragmentation (Heo et al., 2017; Xiong et al., 2014).

It is well known that exercise is vital for health and fitness because it improves immune function, cardiovascular system function, and metabolic function (Gleeson et al., 2011). From a cellular and molecular point of view, chronic exercise training not only promotes mitochondrial function (Gram et al., 2015) and mitochondrial quality control (Konopka et al., 2014; Tarpey et al., 2017) but it also impedes apoptosis (Peterson et al., 2008). For these reasons, studies on the effects of chronic exercise have been mainly carried out at the cellular and molecular level in cardiac and skeletal muscle (Gram et al., 2015; Konopka et al., 2014; Peterson et al., 2008; Tarpey et al., 2017). However, studies on the relationship between a single bout of exercise and apoptosis are insufficient in comparison with those of chronic exercise on cardiac and skeletal muscle. In addition, it is pertinent to examine the effects of a single bout of exercise, since chronic exercise is a successive combination of responses to individual single bouts of exercise. Therefore, this study was performed to investigate the effects of a single bout of exercise on mitochondria-mediated apoptotic signaling in cardiac and skeletal muscle.

\section{MATERIALS AND METHODS}

\section{Experimental design}

Four-month-old Fischer 344 rats were randomly divided into 2 groups ( $\mathrm{n}=10$ per group): control group (CON) and a single bout of exercise group (EX). Cardiac (left ventricle) and skeletal muscles (e.g., soleus, type I fiber and white gastrocnemius, type IIb fiber) were collected from the respective groups and protein levels of mitochondria-mediated apoptotic signaling (e.g., Bax, Bcl-2, cytochrome $\mathrm{c}$, and cleaved caspase-3) were measured via Western immunoblot analysis.

\section{Animals and ethical approval}

All procedures for animal experiments adhered to the stipulations of the National Institutes of Health and the guidelines of the Korean Academy of Medical Science. Rats were housed under controlled environmental conditions with constant illumination (12:12-hr light/dark) and room temperature $\left(20^{\circ} \mathrm{C} \pm 2^{\circ} \mathrm{C}\right)$. Additionally, food and water were made available to the rats ad libitum.

\section{Exercise design}

The treadmill exercise was performed following an adaptation period of about $10 \mathrm{~min}$ for a week. The single bout of EX received a single bout of treadmill exercise training at $20 \mathrm{~m} / \mathrm{min}$ for $1 \mathrm{hr}$. The incline of this exercise was $0 \%$ and the intensity was approximately $\sim 60 \%$ of the rats' maximum oxygen uptake $\left(\mathrm{VO}_{2 \max }\right)$, which corresponds with a moderate intensity (Schefer and Talan, 1996).

\section{Western immunoblot analysis}

The level of proteins involved in mitochondria-mediated apoptotic signaling was determined. Left ventricle, soleus, and white gastrocnemius tissues were collected and frozen immediately at $-80^{\circ} \mathrm{C}$. About $30 \mu \mathrm{g}$ of tissues were homogenized with lysis buffer and centrifuged at 14,000 rpm for $20 \mathrm{~min}$ and $10 \mathrm{~min}$, respectively. Protein levels were quantified by bicinchoninic acid assay method and concentration was confirmed by enzyme-linked immunosorbent assay Reader. Protein was denatured at $95^{\circ} \mathrm{C}$ for $5 \mathrm{~min}$, separated on an sodium dodecyl sulfate-polyacrylamide gel with running buffer at $100 \mathrm{~V}$ for $2 \mathrm{hr}$, and then transferred onto a nitrocellulose membrane with transfer buffer at $170 \mathrm{~mA}$ for $1.5 \mathrm{hr}$. After Ponceau S staining, the membranes were blocked with 5\% skimmed milk in Tris-buffered saline containing 0.1\% Tween-20 at room temperature for $2 \mathrm{hr}$. The membranes were incubated overnight at $4^{\circ} \mathrm{C}$ with the following primary antibodies: GAPDH, Bax, Bcl-2, cytochrome c (Santa Cruz Biotechnology, Dallas, TX, USA) and cleaved caspase-3 (Cell Signaling Technology, Beverly, MA, USA). The membranes that reacted with the primary antibody were incubated at room temperature for $1 \mathrm{hr}$ with horseradish peroxidaseconjugated anti-mouse (Vector Laboratories, Inc., Burlingame, CA, USA) for GAPDH, Bax, Bcl-2, and cytochrome $\mathrm{c}$ and anti-rabbit (Vector Laboratories, Inc.) for cleaved caspase-3. The bands were detected by using the enhanced chemiluminescence detection reagent kit (Thermo Fisher Scientific, Santa Clara, CA, USA) and expressed through Chemidoc (Bio-Rad, Hercules, CA, USA).

\section{Statistical analysis}

Data were presented as mean \pm standard error of the mean. An independent $t$-test was conducted to compare the effects of a single bout of exercise between groups. Significance level was set at 0.05. All graphs were produced using Prism 5 (GraphPad, La Jolla, CA, USA).

\section{RESULTS}

\section{Effects of a single bout of exercise on Bax and Bcl-2 in cardiac and skeletal muscles}

In cardiac and skeletal muscles the levels of Bax, a pro-apoptotic 

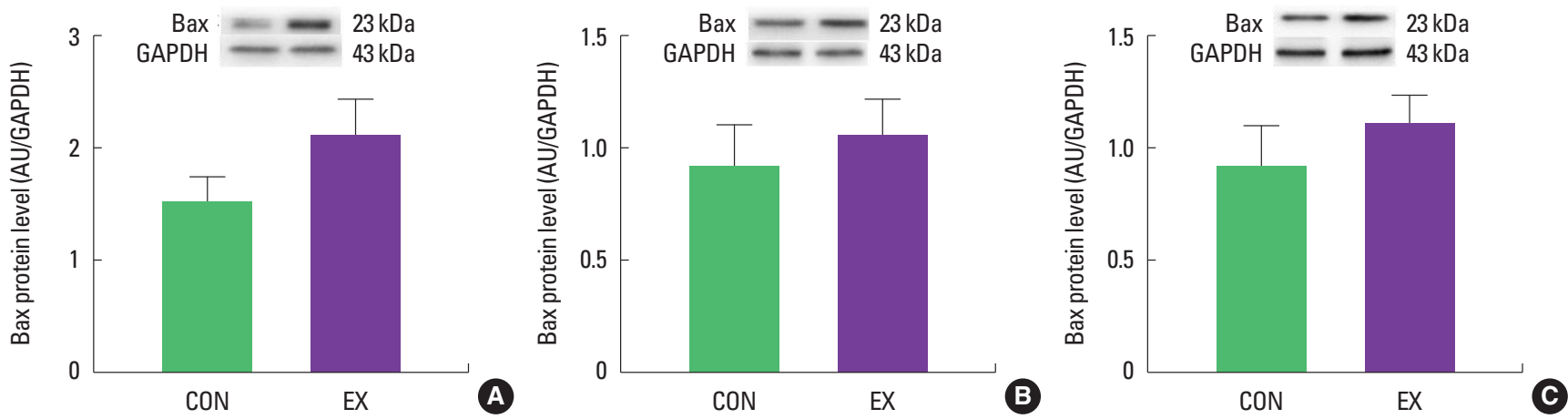

Fig. 1. Effects of a single bout of exercise on Bax protein level in left ventricle (A), soleus (B), and white gastrocnemius (C). The data are shown as mean \pm standard error of the mean. CON, control group; EX, exercise group; AU, arbitrary unit; GAPDH, glyceraldehyde-3-phosphate dehydrogenase.
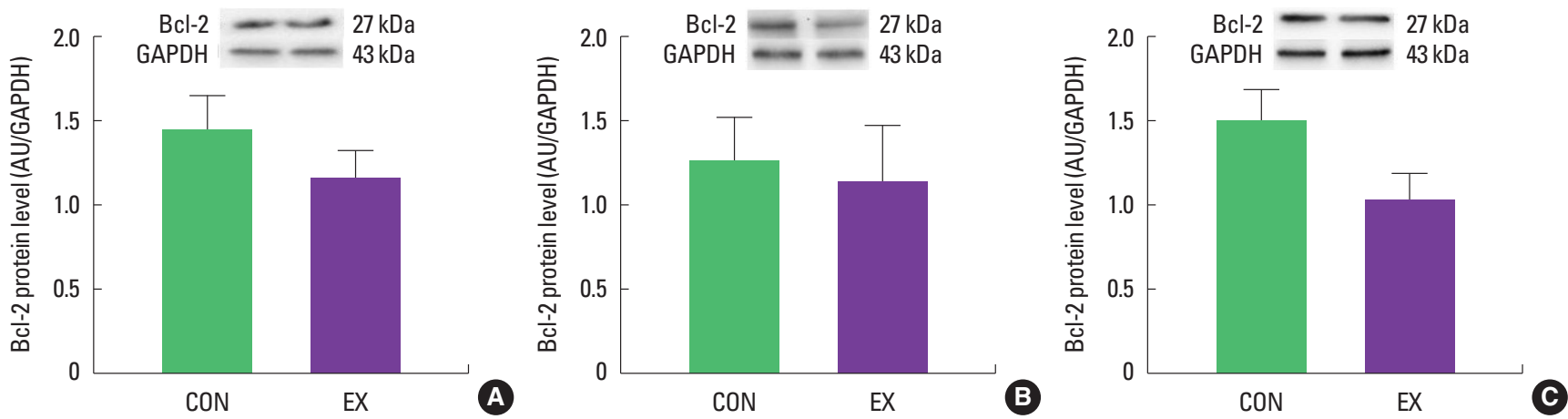

Fig. 2. Effects of a single bout of exercise on Bcl-2 protein level in left ventricle (A), soleus (B), and white gastrocnemius (C). The data are shown as mean \pm standard error of the mean. CON, control group; EX, exercise group; AU, arbitrary unit; GAPDH, glyceraldehyde-3-phosphate dehydrogenase.
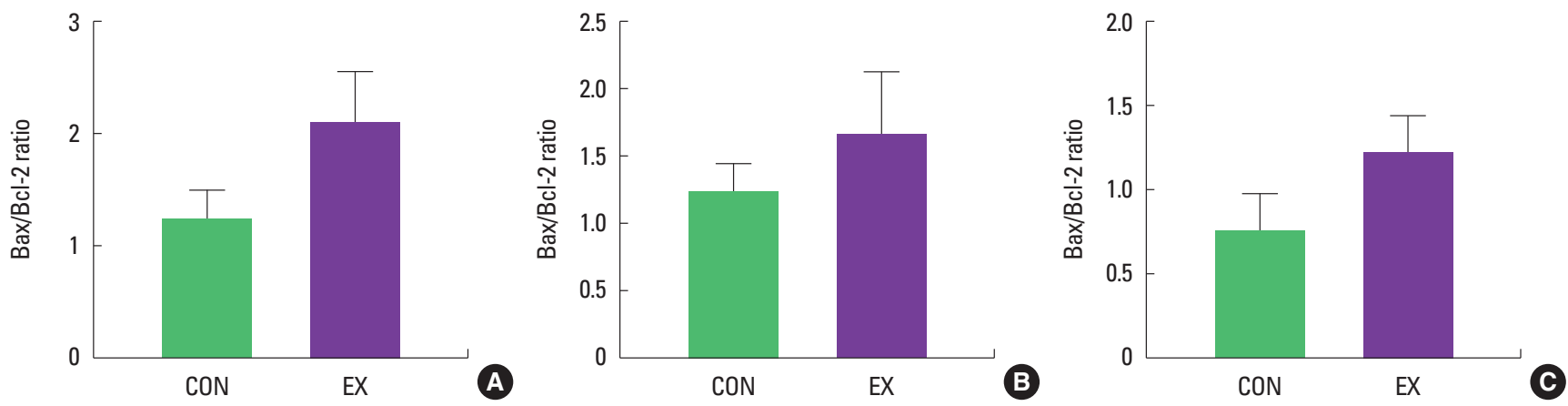

Fig. 3. Effects of a single bout of exercise on Bax/Bcl-2 ratio in left ventricle (A), soleus (B), and white gastrocnemius (C). The data are shown as mean \pm standard error of the mean. $\mathrm{CON}$, control group; EX, exercise group.

protein, were not significantly increased in EX compared with CON (Fig. 1). Also, the levels of Bcl-2, an antiapoptotic protein, were not significantly decreased in EX compared with $\mathrm{CON}$ in all tissues (Fig. 2), suggesting that the Bax/Bcl-2 ratio was not affected by a single bout of exercise (Fig. 3).

\section{Effects of a single bout exercise on mPTP, cytochrome c, and cleaved caspase-3 in cardiac and skeletal muscles}

The mPTP opening sensitivity was significantly decreased in both cardiac and skeletal muscles by a single bout of exercise $(P<$ $0.05)$ (Fig. 4). In contrast, protein levels of cytochrome $c$ and cleaved caspase-3 were not different between CON and EX in cardiac and skeletal muscle (Figs. 5, 6). 

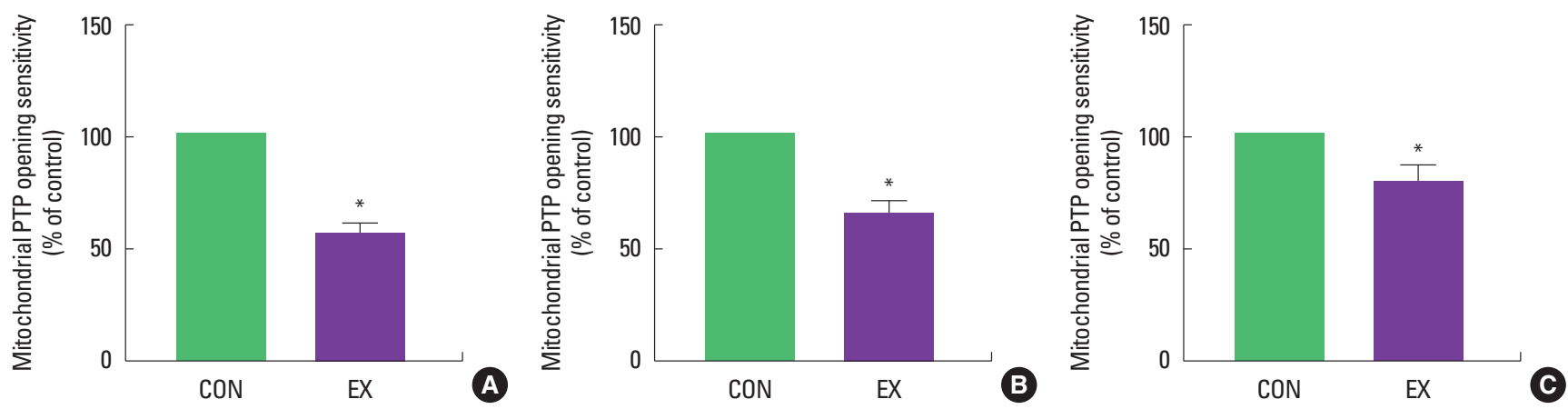

Fig. 4. Effects of a single bout of exercise on mitochondrial permeability transition pore (PTP) opening sensitivity in left ventricle (A), soleus (B), and white gastrocnemius (C). The data are shown as mean \pm standard error of the mean. CON, control group; EX, exercise group; AU, arbitrary unit; GAPDH, glyceraldehyde-3-phosphate dehydrogenase. ${ }^{*} P<0.05$ compared to the control group.
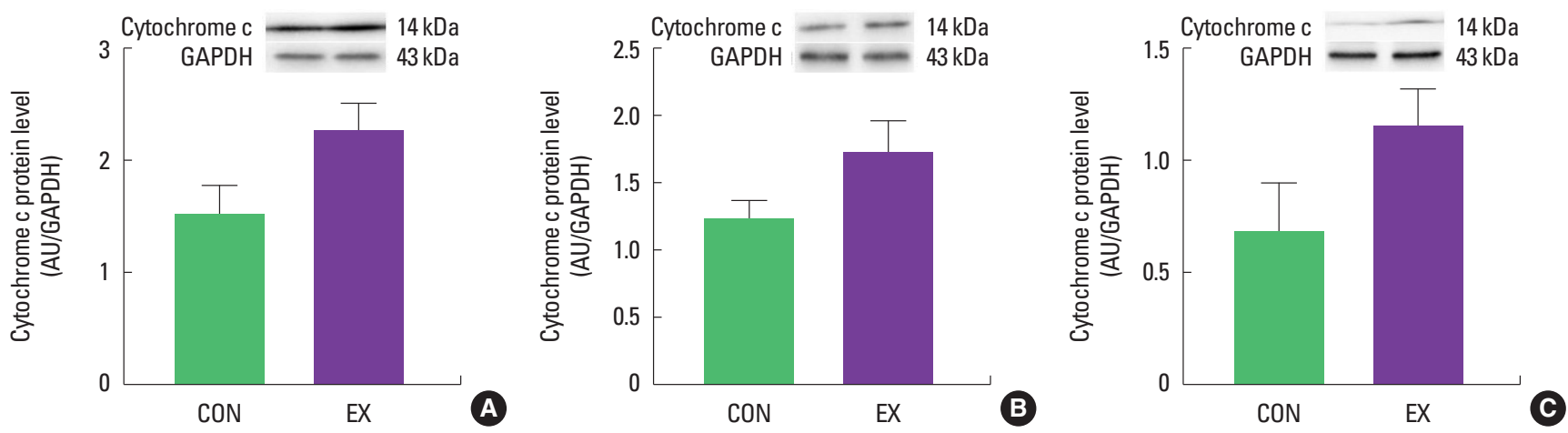

Fig. 5. Effects of a single bout of exercise on cytochrome c protein level in left ventricle (A), soleus (B), and white gastrocnemius (C). The data are shown as mean \pm standard error of the mean. CON, control group; EX, exercise group; AU, arbitrary unit; GAPDH, glyceraldehyde-3-phosphate dehydrogenase.
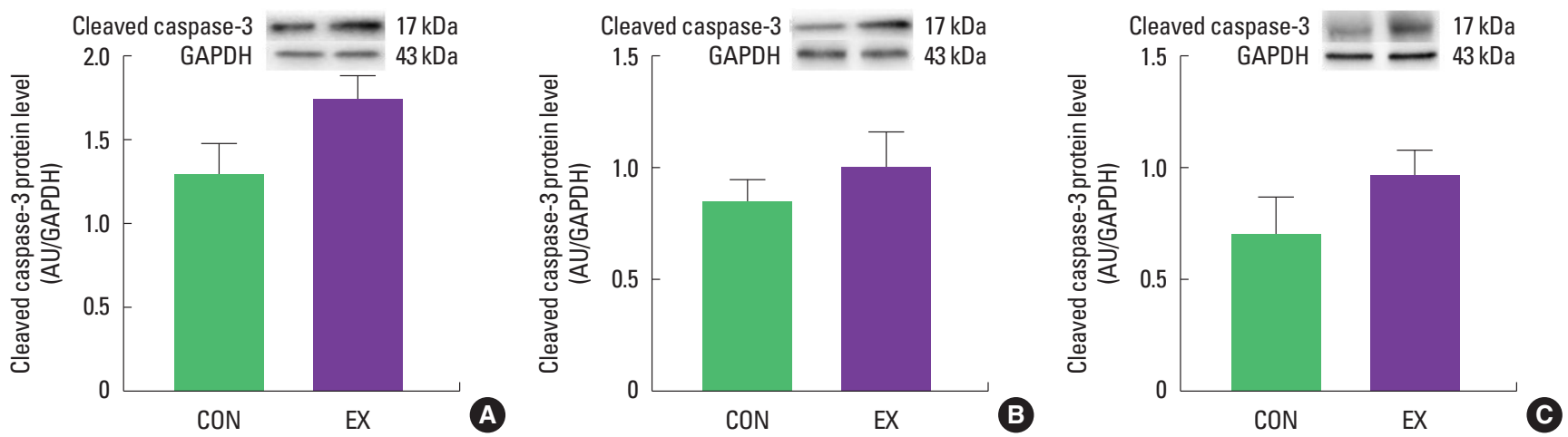

Fig. 6. Effects of a single bout of exercise on cleaved caspase-3 protein level in left ventricle (A), soleus (B), and white gastrocnemius (C). The data are shown as mean \pm standard error of the mean. CON, control group; EX, exercise group; AU, arbitrary unit; GAPDH, glyceraldehyde-3-phosphate dehydrogenase.

\section{DISCUSSION}

The purpose of this study was to investigate mitochondria-mediated apoptotic signaling responses to a single bout of exercise. We observed that a single bout of exercise significantly decreased $\mathrm{mPTP}$ opening sensitivity in all tissues (left ventricle, soleus, and white gastrocnemius). In contrast, a single bout of exercise did not show any statistical differences in Bax, Bcl-2, Bax/Bcl-2 ratio, cytochrome $\mathrm{c}$, and cleaved caspase- 3 between groups in both cardiac and skeletal muscles.

As mentioned before, mitochondria play a vital role in governing cellular events (survival and death) because they coordinate a 
process known as the mitochondria-mediated apoptotic pathway (Quadrilatero et al., 2011). Considering that apoptosis is mainly induced by mtROS and mPTP opening (Redza-Dutordoir and Averill-Bates, 2016; Xiong et al., 2014), our findings lead us to believe that a single bout of exercise may be related to mitochondria-mediated apoptotic signaling, because we observed that mitochondrial $\mathrm{H}_{2} \mathrm{O}_{2}$ emission in the white gastrocnemius (Yoo et al., 2019) was increased and the mPTP opening of all tissues was decreased by a single bout of exercise. mtROS can lead to the release of pro-apoptotic proteins such as Bax into the cytoplasm, consequently inducing increased mPTP opening, caspase-9, cleaved caspase-3, and DNA fragmentation (Heo et al., 2017; Xiong et al., 2014). However, in the current study, a single bout of exercise showed a tendency (a) to increase the protein levels of Bax, cytochrome $\mathrm{c}$, and cleaved caspase-3; (b) to decrease Bcl-2 protein levels; (c) to increase the $\mathrm{Bax} / \mathrm{Bcl}-2$ ratio in all tissues, albeit without statistical significance. The observed data are not in agreement with previous studies. Contrary to our findings, a few studies have reported significant apoptotic markers in relation to a single bout of exercise (Boroujerdi and Rahimi, 2011; Lagranha et al., 2004; Park et al., 2011; Quadrilatero and Hoffman-Goetz, 2005; Rahimi et al., 2018; Sharafi and Rahimi, 2012). Indeed, intense acute resistance exercise resulted in an increase in pro-apoptotic protein, Bax and Bax/Bcl-2 ratio in humans (Rahimi et al., 2018).

Moreover, it was demonstrated that pro-apoptotic proteins increased following strenuous acute exercise in human (Boroujerdi and Rahimi, 2011; Park et al., 2011; Sharafi and Rahimi, 2012) and animal (Lagranha et al., 2004; Quadrilatero and HoffmanGoetz, 2005) studies. We conjecture that these results may be due to the intensity of exercise. The exercise intensity we set in this study is moderate; $~ 60 \%$ of $\mathrm{VO}_{2 \max }$. However, the intensity of exercise utilized in previous studies is high, ranging from $~ 70 \%$ (Park et al., 2011) to 85\% (Lagranha et al., 2004) of $\mathrm{VO}_{2 \max }$. Also, according to Syu et al. (2011), acute severe exercise led to increased oxidative stress and apoptosis but chronic moderate exercise delayed apoptosis. Thus, we believe that a single bout of moderate exercise is insufficient to make a significant difference, but further study is needed to provide a more mechanistic understanding of the apoptosis pathway. One study reported that low intensity but a long duration of acute exhaustive swimming exercise significantly induced an increase in mRNA expression of $\mathrm{Bcl}-2$ and a decrease in activity of caspase-3 (Sun et al., 2016). Moreover, studies of apoptosis in specific tissues are also needed because, while numerous studies have examined blood serum levels, few have also sought to identify apoptosis factors in specific tissues.
In conclusion, a single bout of exercise did not result in observable changes in $\mathrm{Bax}, \mathrm{Bcl}-2, \mathrm{Bax} / \mathrm{Bcl}-2$ ratio, cytochrome $\mathrm{c}$, and cleaved caspase- 3 in cardiac and skeletal muscle. However, a significant decrease in mPTP opening sensitivity in both cardiac and skeletal muscle was observed. Further research is necessary to provide a more mechanistic understanding of mitochondria-mediated apoptosis in the future.

\section{CONFLICT OF INTEREST}

No potential conflict of interest relevant to this article was reported.

\section{ACKNOWLEDGMENTS}

This work was supported by the Inha University Research Grant (2018).

\section{REFERENCES}

Boroujerdi SS, Rahimi R. The apoptotic response to resistance exercise with different intensities in athletes. Med Sport 2011;64:31-44.

Gleeson M, Bishop NC, Stensel DJ, Lindley MR, Mastana SS, Nimmo MA. The anti-inflammatory effects of exercise: mechanisms and implications for the prevention and treatment of disease. Nat Rev Immunol 2011;11:607-615.

Gram M, Vigelsø A, Yokota T, Helge JW, Dela F, Hey-Mogensen M. Skeletal muscle mitochondrial $\mathrm{H}_{2} \mathrm{O}_{2}$ emission increases with immobilization and decreases after aerobic training in young and older men. J Physiol 2015;593:4011-4027.

Heo JW, No MH, Min DH, Kang JH, Kwak HB. Aging-induced sarcopenia and exercise. Asian J Kinesiol 2017;19:43-59.

Konopka AR, Suer MK, Wolff CA, Harber MP. Markers of human skeletal muscle mitochondrial biogenesis and quality control: effects of age and aerobic exercise training. J Gerontol A Biol Sci Med Sci 2014;69: 371-378.

Lagranha CJ, Senna SM, de Lima TM, Silva E, Doi SQ, Curi R, PithonCuri TC. Beneficial effect of glutamine on exercise-induced apoptosis of rat neutrophils. Med Sci Sports Exerc 2004;36:210-217.

Murphy E, Ardehali H, Balaban RS, DiLisa F, Dorn GW 2nd, Kitsis RN, Otsu K, Ping P, Rizzuto R, Sack MN, Wallace D, Youle RJ; American Heart Association Council on Basic Cardiovascular Sciences, Council on Clinical Cardiology, and Council on Functional Genomics and Translational Biology. Mitochondrial function, biology, and role in disease: a scientific statement from the American Heart Association. 
Circ Res 2016;118:1960-1991.

Park KS, Sedlock DA, Navalta JW, Lee MG, Kim SH. Leukocyte apoptosis and pro-/anti-apoptotic proteins following downhill running. Eur J Appl Physiol 2011;111:2349-2357.

Peterson JM, Bryner RW, Sindler A, Frisbee JC, Alway SE. Mitochondrial apoptotic signaling is elevated in cardiac but not skeletal muscle in the obese Zucker rat and is reduced with aerobic exercise. J Appl Physiol (1985) 2008;105:1934-1943.

Quadrilatero J, Alway SE, Dupont-Versteegden EE. Skeletal muscle apoptotic response to physical activity: potential mechanisms for protection. Appl Physiol Nutr Metab 2011;36:608-617.

Quadrilatero J, Hoffman-Goetz L. N-acetyl-l-cysteine protects intestinal lymphocytes from apoptotic death after acute exercise in adrenalectomized mice. Am J Physiol Regul Integr Comp Physiol 2005;288:R1664R1672.

Rahimi MR, Khabiri P, Faraji H. Effects of caffeine ingestion on resistance exercise-induced apoptosis in athletes: a randomized, double-blind, placebocontrolled, crossover study. Prog Nut 2018;20:563-569.

Redza-Dutordoir M, Averill-Bates DA. Activation of apoptosis signalling pathways by reactive oxygen species. Biochim Biophys Acta 2016;1863: 2977-2992.

Schefer V, Talan MI. Oxygen consumption in adult and AGED C57BL/6J mice during acute treadmill exercise of different intensity. Exp Gerontol 1996;31:387-392.

Sharafi H, Rahimi R. The effect of resistance exercise on p53, caspase-9, and caspase-3 in trained and untrained men. J Strength Cond Res 2012; 26:1142-1148.

Sun Y, Cui D, Zhang Z, Zhang T, Shi J, Jin H, Ge Z, Ji L, Ding S. Attenuated oxidative stress following acute exhaustive swimming exercise was accompanied with modified gene expression profiles of apoptosis in the skeletal muscle of mice. Oxid Med Cell Longev 2016;2016:8381242.

Syu GD, Chen HI, Jen CJ. Severe exercise and exercise training exert opposite effects on human neutrophil apoptosis via altering the redox status. PLoS One 2011;6:e24385.

Tarpey MD, Davy KP, McMillan RP, Bowser SM, Halliday TM, Boutagy NE, Davy BM, Frisard MI, Hulver MW. Skeletal muscle autophagy and mitophagy in endurance-trained runners before and after a highfat meal. Mol Metab 2017;6:1597-1609.

Xiong S, Mu T, Wang G, Jiang X. Mitochondria-mediated apoptosis in mammals. Protein Cell 2014;5:737-749.

Yoo SZ, No MH, Heo JW, Park DH, Kang JH, Kim JH, Seo DY, Han J, Jung SJ, Kwak HB. Effects of acute exercise on mitochondrial function, dynamics, and mitophagy in rat cardiac and skeletal muscles. Int Neurourol J 2019;23(Suppl 1):S22-31. 